\title{
EXISTENCE AND MULTIPLICITY OF SOLUTIONS FOR A KIRCHHOFF SYSTEM WITH CRITICAL GROWTH
}

\author{
Marcelo F. Furtado, Luan D. de Oliveira and João Pablo P. da Silva \\ Universidade de Brasília, Departamento de Matemática \\ 70910-900, Brasília-DF, Brazil; mfurtado@unb.br \\ Universidade de Brasília, Departamento de Matemática \\ 70910-900, Brasília-DF, Brazil; luandiego2000@hotmail.com \\ Universidade Federal do Pará, Departamento de Matemática \\ 66075-110, Belém-PA, Brazil; jpabloufpa@gmail.com
}

Abstract. We consider the system

$$
\begin{cases}-m\left(\|u\|^{2}\right) \Delta u=\lambda F_{u}(x, u, v)+\frac{1}{2^{*}} G_{u}(u, v), & \text { in } \Omega, \\ -l\left(\|v\|^{2}\right) \Delta v=\lambda F_{v}(x, u, v)+\frac{1}{2^{*}} G_{v}(u, v), & \text { in } \Omega, \\ u, v \in H_{0}^{1}(\Omega) & \end{cases}
$$

where $\Omega \subset \mathbf{R}^{N}, N \geq 3$, is a bounded smooth domain, $\|\cdot\|^{2}=\int_{\Omega}|\nabla \cdot|^{2} \mathrm{~d} x, \lambda>0$ is a parameter, the functions $m$ and $l$ are positive and increasing, the function $F$ is superlinear both at origin and at infinity, the function $G$ is $2^{*}$-homogeneous. In our first result, we obtain a nonzero nonnegative solution for large values of $\lambda$. We also prove that, for any $k \in \mathbf{N}$, there exists $\lambda_{k}^{*}>0$ such that the problem has at least $k$ pairs of nonzero solutions if $\lambda \geq \lambda_{k}^{*}$.

\section{Introduction}

Given a bounded smooth domain $\Omega \subset \mathbf{R}^{N}$ and a positive function $m$, the Kirchhoff equation

$$
-m\left(\int_{\Omega}|\nabla u|^{2} \mathrm{~d} x\right) \Delta u=g(x, u), \text { in } \Omega, \quad u=0, \text { on } \partial \Omega,
$$

has its origin in the theory of nonlinear vibration. For instance, in the case $m(t)=$ $a+b t$, with $a, b>0$, it comes from the following model for the modified d'Alembert wave equation

$$
\rho \frac{\partial^{2} u}{\partial t^{2}}-\left(\frac{P_{0}}{h}+\frac{E}{2 L} \int_{0}^{L}\left|\frac{\partial u}{\partial x}\right|^{2} d x\right) \frac{\partial^{2} u}{\partial x^{2}}=g(x, u),
$$

proposed by Kirchhoff in [12]. Its main feature is to consider the effects of the changes on the length of the string during vibrations. The parameters have the following meaning: $L$ is the length of the string, $h$ is the area of cross-section, $E$ is the Young modulus of the material, $\rho$ is the mass density and $P_{0}$ is the initial tension. Nonlocal problems also appear in other fields as, for example, biological systems where $u$ describes a process which depends on the average of itself (for instance, population density). We refer the reader to $[4,15,14]$ and their references for more examples on the physical motivation of this problem.

https://doi.org/10.5186/aasfm.2021.4620

2020 Mathematics Subject Classification: Primary 35J60; Secondary 35J20.

Key words: Kirchhoff-type problems, multiple solutions, critical nonlinearities.

The first author was partially supported by $\mathrm{CNPq} /$ Brazil and FAP-DF/Brazil. 
In this paper, we are interested in the nonlocal system

$$
\begin{cases}-m\left(\int_{\Omega}|\nabla u|^{2} \mathrm{~d} x\right) \Delta u=\lambda F_{u}(x, u, v)+\frac{1}{2^{*}} G_{u}(u, v) & \text { in } \Omega \\ -l\left(\int_{\Omega}|\nabla v|^{2} \mathrm{~d} x\right) \Delta v=\lambda F_{v}(x, u, v)+\frac{1}{2^{*}} G_{v}(u, v) & \text { in } \Omega \\ u, v \in H_{0}^{1}(\Omega), & \end{cases}
$$

where $\Omega \subset \mathbf{R}^{N}, N \geq 3$, is a bounded smooth domain, $\lambda>0$ is a parameter, $2^{*}:=2 N /(N-2)$ and the functions $m, l$ satisfy

$\left(m_{0}\right) m:[0,+\infty] \rightarrow \mathbf{R}$ is continuous, nondecreasing in $\left[0, \sigma_{m}\right]$ and $m(0)>0$;

$\left(l_{0}\right) l:[0,+\infty] \rightarrow \mathbf{R}$ is continuous, nondecreasing in $\left[0, \sigma_{l}\right]$ and $l(0)>0$.

We are denoting by $F_{u}$ and $F_{v}$ the partial derivatives with respect to the second and third variable, respectively, of the nonlinearity $F: \Omega \times \mathbf{R}^{2} \rightarrow \mathbf{R}$. The assumptions on $F$ are:

$\left(F_{0}\right) F \in C^{1}\left(\Omega \times \mathbf{R}^{2}, \mathbf{R}\right)$;

$\left(F_{1}\right)$ there exists $q \in\left(2,2^{*}\right)$ such that

$$
\lim _{|z| \rightarrow \infty} \frac{|\nabla F(x, z)|}{|z|^{q-1}}=0, \quad \text { uniformly in } \Omega
$$

$\left(F_{2}\right)$ there exists $\theta \in\left(2,2^{*}\right)$ such that

$$
0 \leq \theta F(x, z) \leq z \cdot \nabla F(x, z), \quad \forall z \in \mathbf{R}^{2},
$$

where $z_{1} \cdot z_{2}$ denotes the inner product of $z_{1}, z_{2} \in \mathbf{R}^{2}$;

$\left(F_{3}\right)$ there holds

$$
\lim _{|z| \rightarrow 0} \frac{|\nabla F(x, z)|}{|z|}=0, \quad \text { uniformly in } \Omega
$$

$\left(F_{4}\right) F_{u}(x, 0, t)=0, F_{v}(x, s, 0)=0$, for any $(s, t) \in \mathbf{R}^{2}$.

Setting $\mathbf{R}_{+}^{2}:=\left\{(s, t) \in \mathbf{R}^{2}: s \geq 0, t \geq 0\right\}$, the hypotheses for $G: \mathbf{R}^{2} \rightarrow \mathbf{R}$ are the following:

$\left(G_{0}\right) G \in C^{1}\left(\mathbf{R}^{2}, \mathbf{R}\right)$ is $2^{*}$-homogeneous, that is,

$$
G(\sigma s, \sigma t)=\sigma^{2^{*}} G(s, t), \quad \forall \sigma>0,(s, t) \in \mathbf{R}^{2} ;
$$

$\left(G_{1}\right) G(s, t)>0$, for any $(s, t) \in \mathbf{R}_{+}^{2} \backslash\{(0,0)\}$;

$\left(G_{2}\right)$ one of the conditions below holds:

(a) $G_{u}(0,1)=0, G_{v}(1,0)=0$

(b) $G_{u}(0,1)>0, G_{v}(1,0)>0$.

From now on, we denote by $H$ the Hilbert space $H_{0}^{1}(\Omega) \times H_{0}^{1}(\Omega)$ endowed with the norm

$$
\|(u, v)\|:=\left[\int_{\Omega}\left(|\nabla u|^{2}+|\nabla v|^{2}\right) \mathrm{d} x\right]^{1 / 2} .
$$

For each component of the vector $(u, v)$, we also denote $\|\cdot\|=\left(\int_{\Omega}|\nabla \cdot|^{2}\right)^{1 / 2}$.

We say that a solution $(u, v) \in H$ of the system $\left(S_{\lambda}\right)$ is nonnegative if each component is a nonnegative function. In our first, result we obtain the existence of such kind of solution if $\lambda$ is large:

Theorem 1.1. Suppose that $m, l, F$ and $G$ satisfy $\left(m_{0}\right),\left(l_{0}\right),\left(F_{0}\right)-\left(F_{4}\right)$ and $\left(G_{0}\right)-\left(G_{2}\right)$. Then there exists $\lambda^{*}>0$ such that, for any $\lambda>\lambda^{*}$, problem $\left(S_{\lambda}\right)$ has a nonzero nonnegative solution $\left(u_{\lambda}, v_{\lambda}\right) \in H$ such that $\left\|\left(u_{\lambda}, v_{\lambda}\right)\right\| \rightarrow 0$ as $\lambda \rightarrow+\infty$. 
In the proof, we apply the Moutain Pass Theorem. The technical conditions $\left(F_{4}\right),\left(G_{1}\right)-\left(G_{2}\right)$ are used only to verify that the critical points of the energy functional associated with $\left(S_{\lambda}\right)$ have nonnegative components (see [19]). For dimensions $N \geq 4$, the critical exponent $2^{*}$ is smaller than or equal to 4 , and therefore the integral $\int_{\Omega} G(u, v) \mathrm{d} x$ does not dominate the fourth-order term which comes from the nonlocal part of the system. In order to overcome this difficulty, we follow the procedure introduced in [1]. It consists in dealing with a truncated problem and, after solving it, verify that the obtained solutions have small norm and therefore solve the original problem.

In our second result, we replace the conditions $\left(F_{4}\right),\left(G_{1}\right),\left(G_{2}\right)$ by the following:

$\left(F_{5}\right) F(x, \cdot)$ is even;

$\left(\widehat{G_{1}}\right) G(s, t) \geq 0$, for any $(s, t) \in \mathbf{R}^{2}$;

$\left(G_{3}\right) G$ is even.

We prove the following:

Theorem 1.2. Suppose that $m, l, F$ and $G$ satisfy $\left(m_{0}\right),\left(l_{0}\right),\left(F_{0}\right)-\left(F_{3}\right),\left(F_{5}\right)$, $\left(G_{0}\right),\left(\widehat{G_{1}}\right),\left(G_{3}\right)$. Then, for any $k \in \mathbf{N}$, there exists $\lambda_{k}^{*}>0$ such that problem $\left(S_{\lambda}\right)$ has $k$ pairs of nonzero solutions for any $\lambda \geq \lambda_{k}^{*}$.

Under conditions $\left(F_{5}\right)$ and $\left(G_{3}\right)$, the energy functional is even. Hence, we are able to apply the Symmetric Mountain Pass Theorem to get multiple solutions. Differently from Theorem 1.1, we have no information about the sign of such solutions.

As far as we know, the first paper dealing with Kirchhoff type equation via variational methods was [1]. By assuming some technical conditions on $m$ and the nonlinearity, they obtained a solution for the scalar version of the problem $\left(S_{\lambda}\right)$. Since then, there is a vast literature concerning existence, nonexistence, multiplicity and concentration behavior of solutions for nonlocal problems. We just quote $[10,18$, $11,17,22]$ for subcritical problems and $[9,7,20,13]$ for the critical ones. The main results of this paper extend and complement that of $[6,8,5,3]$ in several senses: we consider system of equations, we deal with different functions and higher dimensions and we prove a multiplicity result. Moreover, differently from the aforementioned works, our hypotheses on $m$ and $l$ are only near the origin.

Before finishing the introduction we present some examples. Given $l>0$, we set

$$
P_{l}(s, t):=a_{1}|s|^{l}+a_{2}|t|^{l}+\sum_{i=1}^{k} b_{i}|s|^{\alpha_{i}}|t|^{\beta_{i}},
$$

with $\alpha_{i}, \beta_{i}>1, \alpha_{i}+\beta_{i}=l$ and $a_{1}, a_{2}, b_{i} \in \mathbf{R}$. The following functions and your combinations, with appropriated choices of the constants, verify our assumptions on the critical homogeneous nonlinearity $G$ :

$$
G(s, t)=P_{2^{*}}(s, t), \quad G(s, t)=\sqrt[r]{P_{r 2^{*}}(s, t)}, \quad G(s, t)=\frac{P_{2^{*}+r}(s, t)}{P_{r}(s, t)},
$$

with $r>0$. Although the same prototype can be used for $F$, with $2^{*}$ replaced by $q_{0}$ verifying $2<q_{0}<q<2^{*}$, we notice that $F$ can also be nonhomogeneous.

The rest of this paper is organized as follows: in the next section, we present the variational setting to deal with $\left(S_{\lambda}\right)$. Section 3 is devoted to the proof of local compactness condition for the energy functional. We prove our main theorems in Sections 4 and 5 . 


\section{Variational setting and technical results}

In what follows we write $\int_{\Omega} u$ instead of $\int_{\Omega} u(x) \mathrm{d} x$. We denote by $\|u\|_{p}$ the $L^{p}(\Omega)$-norm of a function $u \in L^{p}(\Omega)$, for any $1 \leq p \leq \infty$. Throughout the paper we shall assume that conditions $\left(m_{0}\right),\left(l_{0}\right),\left(F_{0}\right)-\left(F_{3}\right)$ and $\left(G_{0}\right)$ hold. We write $o_{n}(1)$ to denote a quantity approaching zero as $n \rightarrow+\infty$.

Let $\theta>2$ be given by $\left(F_{2}\right)$. Using conditions $\left(m_{0}\right)$ and $\left(l_{0}\right)$, we can find $s_{0} \in$ $\left(0, \sigma_{m}\right)$ and $s_{1} \in\left(0, \sigma_{l}\right)$ such that, for

$$
a:=m\left(s_{0}\right), \quad b:=l\left(s_{1}\right),
$$

there hold

$$
m(0) \leq a<\frac{\theta}{2} m(0), \quad l(0) \leq b<\frac{\theta}{2} l(0) .
$$

Define the functions $m_{a}, l_{b} \in C\left(\mathbf{R}^{+}, \mathbf{R}^{+}\right)$by

$$
m_{a}(s):=\left\{\begin{array}{ll}
m(s), & \text { if } 0 \leq s \leq s_{0}, \\
a, & \text { if } s \geq s_{0},
\end{array} \quad l_{b}(s):= \begin{cases}l(s), & \text { if } 0 \leq s \leq s_{1}, \\
b, & \text { if } s \geq s_{1},\end{cases}\right.
$$

and consider from now on the modified problem

$$
\begin{cases}-m_{a}\left(\|u\|^{2}\right) \Delta u=\lambda F_{u}(x, u, v)+\frac{1}{2^{*}} G_{u}(u, v), & \text { in } \Omega, \\ -l_{b}\left(\|v\|^{2}\right) \Delta v=\lambda F_{v}(x, u, v)+\frac{1}{2^{*}} G_{v}(u, v), & \text { in } \Omega, \\ u, v \in H_{0}^{1}(\Omega) . & \end{cases}
$$

Since in our first result we are looking for nonnegative solutions, without loss of generality we may suppose that

$$
\begin{cases}F_{u}(x, s, t)=0, & \text { if } x \in \Omega, s \leq 0, t \in \mathbf{R}, \\ F_{v}(x, s, t)=0, & \text { if } x \in \Omega, s \in \mathbf{R}, t \leq 0 .\end{cases}
$$

By $\left(F_{4}\right)$, the above assumptions do not affect the continuity of $F_{u}$ and $F_{v}$. Moreover, by using $\left(F_{0}\right),\left(F_{1}\right)$ and $\left(F_{3}\right)$, we obtain after integration

$$
|F(x, z)| \leq \frac{\varepsilon}{2}|z|^{2}+C|z|^{q}, \quad \forall x \in \Omega, z \in \mathbf{R}^{2},
$$

and therefore the functional $(u, v) \mapsto \int_{\Omega} F(x, u, v)$ is well defined. Moreover, by using $\left(F_{1}\right)$ again, we can check that it belongs to $C^{1}(H, \mathbf{R})$.

Lemma 2.1. Suppose that $G$ satisfies $\left(G_{2}\right)$. Then there exists $\widetilde{G} \in C^{1}\left(\mathbf{R}^{2}, \mathbf{R}^{+}\right)$ such that $\widetilde{G}$ satisfies $\left(G_{0}\right)-\left(G_{1}\right)$ and $\widetilde{G} \equiv G$ in $\mathbf{R}^{+} \times \mathbf{R}^{+}$. Moreover, if $\left(G_{2}\right)(b)$ holds, then

$$
\left\{\begin{array}{l}
\widetilde{G}_{u}(s, t) \geq 0, \quad \text { if } s \leq 0, t \in \mathbf{R}, \\
\widetilde{G}_{v}(s, t) \geq 0, \quad \text { if } s \in \mathbf{R}, t \leq 0 .
\end{array}\right.
$$

Proof. Since $G$ is $2^{*}$-homogeneous, its gradient is $\left(2^{*}-1\right)$-homogeneous and, for any $(s, t) \in \mathbf{R}^{2}$, there hold

$$
(s, t) \cdot \nabla G(s, t)=2^{*} G(s, t)
$$

and

$$
|G(s, t)| \leq M_{G}\left(|s|^{2^{*}}+|t|^{2^{*}}\right),
$$


where $M_{G}:=\max \left\{G(s, t): s, t \in \mathbf{R},|s|^{2^{*}}+|t|^{2^{*}}=1\right\}$. Hence, we can check that the lemma holds for the function

$$
\widetilde{G}(s, t)= \begin{cases}G\left(s^{+}, t^{+}\right), & \text {if }\left(G_{2}\right)(a) \text { holds, } \\ G\left(s^{+}, t^{+}\right)-\left(s^{-}, t^{-}\right) \cdot \nabla G\left(s^{+}, t^{+}\right), & \text {if }\left(G_{2}\right)(b) \text { holds }\end{cases}
$$

defined for $(s, t) \in \mathbf{R}^{2}$. We omit the details.

We are ready to introduce the energy functional associated with the modified problem, namely $I_{a, b, \lambda}: H \rightarrow \mathbf{R}$ given by

$$
I_{a, b, \lambda}(u, v):=\frac{1}{2} M_{a}\left(\|u\|^{2}\right)+\frac{1}{2} L_{b}\left(\|v\|^{2}\right)-\lambda \int_{\Omega} F(x, u, v)-\frac{1}{2^{*}} \int_{\Omega} \widetilde{G}(u, v),
$$

with $M_{a}(s):=\int_{0}^{s} m_{a}(t) d t$ and $L_{b}(s):=\int_{0}^{s} l_{b}(t) \mathrm{d} t$. It is standard to check that $I_{a, b, \lambda} \in C^{1}(H, \mathbf{R})$.

In order to simplify the notation, in the next two sections, we are going to write $G$ to denote the function $\widetilde{G}$ given by the last lemma. Since $\widetilde{G}$ satisfies $\left(G_{0}\right)-\left(G_{1}\right)$ this can be done without loss of generality. Moreover, we have the following:

Lemma 2.2. Suppose that $(u, v) \in H$ is such that $I_{a, b, \lambda}^{\prime}(u, v)=0,\|u\|<s_{0}$ and $\|v\|<s_{1}$, where $s_{0}, s_{1}$ come from (2.1). Then $(u, v)$ is a nonnegative solution of problem $\left(S_{\lambda}\right)$.

Proof. It is clear that $(u, v)$ is a weak solution of the modified problem $\left(\widehat{S}_{\lambda}\right)$. If $(u, v)$ is nonnegative then, since $\|u\|<s_{0}$ and $\|v\|<s_{1}$, it follows from the definition of $m_{a}$ and $l_{b}$ that $m_{a}\left(\|u\|^{2}\right)=m\left(\|u\|^{2}\right)$ and $l_{b}\left(\|v\|^{2}\right)=l\left(\|u\|^{2}\right)$. Hence, $(u, v)$ is a nonnegative solution of $\left(S_{\lambda}\right)$.

In order to check that $(u, v)$ is nonnegative, we first notice that, by $(2.3)$,

$$
\int_{\Omega}\left(u^{-}, 0\right) \cdot \nabla F(x, u, v)=\int_{\{u \geq 0\}} u^{-} F_{u}(x, u, v) \mathrm{d} x+\int_{\{u<0\}} u^{-} F_{u}(x, u, v) \mathrm{d} x=0,
$$

where $u^{+}(x):=\max \{u(x), 0\}$ and $u^{-}:=u^{+}-u$. Moreover, if $J(u, v):=\int_{\Omega} G(u, v)$ and $\left(G_{2}\right)(a)$ holds, it follows from (2.6) that

$$
\left\langle J^{\prime}(u, v),\left(u^{-}, 0\right)\right\rangle=\int_{\Omega} u^{-} G_{u}\left(u^{+}, v^{+}\right)=\int_{\{u \leq 0\}} u^{-} G_{u}\left(0, v^{+}\right) \mathrm{d} x=0,
$$

since $G_{u}(0,1)=0$. If $\left(G_{2}\right)(b)$ holds, we can argue as above and use Lemma 2.1 to get

$$
\left\langle J^{\prime}(u, v),\left(u^{-}, 0\right)\right\rangle=\int_{\{u \leq 0\}} u^{-} G_{u}(u, v) \mathrm{d} x \geq 0,
$$

and therefore $\left\langle J^{\prime}(u, v),\left(u^{-}, 0\right)\right\rangle \geq 0$, whenever $\left(G_{2}\right)$ holds.

The above considerations together with $\left\langle I_{a, b, \lambda}^{\prime}(u, v),\left(u^{-}, 0\right)\right\rangle=0$ imply that

$$
0 \leq-m_{a}\left(\|u\|^{2}\right) \int_{\Omega}\left|\nabla u^{-}\right|^{2}
$$

and therefore we conclude that $u \geq 0$ a.e. in $\Omega$. Repeating the argument and using $\left\langle I_{a, b, \lambda}^{\prime}(u, v),\left(0, v^{-}\right)\right\rangle=0$, we also get $v \geq 0$ a.e. in $\Omega$.

We present now some technical results. Following [19], we introduce the number

$$
S_{G}:=\inf _{(u, v) \in H \backslash\{(0,0)\}} \frac{\int_{\Omega}\left(|\nabla u|^{2}+|\nabla v|^{2}\right)}{\left(\int_{\Omega} G(u, v)\right)^{2 / 2^{*}}} .
$$


We denote by $C(\bar{\Omega})$ the set of all continuos functions $u: \bar{\Omega} \rightarrow \mathbf{R}$ endowed with the norm $\|u\|_{C(\bar{\Omega})}:=\max _{x \in \bar{\Omega}}|u(x)|$ and by $\mathcal{M}(\bar{\Omega})$ its dual, namely the set of Radon measures. The next result is a variant of the classical concentration-compactness lemma of Lions [16, Lemma 1.1]. It was inspired by an earlier version presented in [19, Lemma 6] and its proof can de done with natural changes in the arguments presented in this last paper.

Lemma 2.3. Suppose that $\left(\left(u_{n}, v_{n}\right)\right) \subset H$ is such that

$$
\begin{cases}\left(u_{n}, v_{n}\right) \rightarrow(u, v), & \text { weakly in } H, \\ \left(\left|\nabla u_{n}\right|^{2}+\left|\nabla v_{n}\right|^{2}\right) \rightarrow \zeta, & \text { in the weak- } \text { topology } \sigma(\mathcal{M}(\bar{\Omega}), C(\bar{\Omega})), \\ G\left(u_{n}, v_{n}\right) \rightarrow \nu, & \text { in the weak- topology } \sigma(\mathcal{M}(\bar{\Omega}), C(\bar{\Omega})),\end{cases}
$$

where $\zeta, \nu \in \mathcal{M}(\bar{\Omega})$ are nonnegative bounded measures over $\bar{\Omega}$. Then there exist an enumerable set $J$, which can be empty, a family of distinct points $\left(x_{j}\right)_{j \in J} \subset \bar{\Omega}$, and $\left(\nu_{j}\right)_{j \in J},\left(\zeta_{j}\right)_{j \in J} \subset(0,+\infty)$ such that

(a) $\nu=G(u, v)+\sum_{j \in J} \nu_{j} \delta_{x_{j}}$

(b) $\zeta \geq\left(|\nabla u|^{2}+|\nabla v|^{2}\right)+\sum_{j \in J} \zeta_{j} \delta_{x_{j}}$,

where $\delta_{x}$ indicates the Dirac mass at $x$. Moreover, for any $j \in J$, we have that $S_{G} \nu_{j}^{2 / 2^{*}} \leq \zeta_{j}$

We prove in the sequel that, for some special sequences, the set $J$ is finite.

Lemma 2.4. Let $\left(z_{n}\right) \subset H$ be as in Lemma 2.3 and suppose that $I_{a, b, \lambda}^{\prime}\left(z_{n}\right) \rightarrow 0$, as $n \rightarrow \infty$. Then $J$ has a finite number of elements. Moreover,

$$
\nu_{j} \geq\left(\min \{m(0), l(0)\} S_{G}\right)^{N / 2}, \quad \forall j \in J .
$$

Proof. Let $\phi \in C_{0}^{\infty}\left(\mathbf{R}^{N},[0,1]\right)$ be such that $\phi \equiv 1$ in $B_{1 / 2}(0)$ and $\phi \equiv 0$ in $\mathbf{R}^{N} \backslash B_{1}(0)$. Suppose that $J \neq \varnothing$, fix $j \in J$ and define $\phi_{\varepsilon}(x):=\phi\left(\left(x-x_{j}\right) / \varepsilon\right)$, for $\varepsilon>0$. Since $\left(\phi_{\varepsilon} z_{n}\right) \subset H$ is bounded, we have that $\left\langle I_{a, b, \lambda}^{\prime}\left(z_{n}\right),\left(\phi_{\varepsilon} z_{n}\right)\right\rangle=o_{n}(1)$. This and (2.4) provide

$$
\begin{aligned}
o_{n}(1)= & m_{a}\left(\left\|u_{n}\right\|^{2}\right)\left(A_{n, \varepsilon}+\int_{\Omega}\left|\nabla u_{n}\right|^{2} \phi_{\varepsilon}\right)+l_{b}\left(\left\|v_{n}\right\|^{2}\right)\left(B_{n, \varepsilon}+\int_{\Omega}\left|\nabla v_{n}\right|^{2} \phi_{\varepsilon}\right) \\
& -\int_{\Omega} \phi_{\varepsilon} G\left(u_{n}, v_{n}\right)-\lambda \int_{\Omega}\left(\phi_{\varepsilon} z_{n}\right) \cdot \nabla F\left(x, z_{n}\right) .
\end{aligned}
$$

where

$$
A_{n, \varepsilon}:=\int u_{n}\left(\nabla u_{n} \cdot \nabla \phi_{\varepsilon}\right), \quad B_{n, \varepsilon}:=\int v_{n}\left(\nabla v_{n} \cdot \nabla \phi_{\varepsilon}\right) .
$$

Since $F$ has subcritical growth, we can use Sobolev embeddings and Lebesgue Theorem to get

$$
\lim _{n \rightarrow+\infty} \int_{\Omega}\left(\phi_{\varepsilon} z_{n}\right) \cdot \nabla F\left(x, z_{n}\right)=\int_{\Omega}\left(\phi_{\varepsilon} z\right) \cdot \nabla F(x, z),
$$

where $z=(u, v)$ is the weak limit of $\left(z_{n}\right)$ in $H$. Recalling that $m_{a}(s) \geq m(0)$ and $l_{b}(s) \geq l(0)$, we can use $(2.8)$ and Lemma 2.3 to get

$$
\min \{m(0), l(0)\}\left(A_{n, \varepsilon}+B_{n, \varepsilon}+\int_{\Omega} \phi_{\varepsilon} \mathrm{d} \zeta\right) \leq \int_{\Omega} \phi_{\varepsilon} \mathrm{d} \nu+\lambda \int_{\Omega}\left(\phi_{\varepsilon} z\right) \cdot \nabla F(x, z)
$$


We claim that

$$
\lim _{\varepsilon \rightarrow 0} \limsup _{n \rightarrow \infty} A_{n, \varepsilon}=0, \quad \lim _{\varepsilon \rightarrow 0} \limsup _{n \rightarrow \infty} B_{n, \varepsilon}=0 .
$$

If this is true, we can take the limit as $n \rightarrow+\infty, \varepsilon \rightarrow 0$ in (2.9) and use Lebesgue Theorem to conclude that

$$
\min \{m(0), l(0)\} \zeta_{j} \leq \nu_{j}
$$

Recalling that $S_{G} \nu_{j}^{2 / 2^{*}} \leq \zeta_{j}$, we obtain

$$
\min \{m(0), l(0)\} S_{G} \nu_{j}^{2 / 2^{*}} \leq \min \{m(0), l(0)\} \zeta_{j} \leq \nu_{j}
$$

and therefore $\nu_{j} \geq\left[\min \{m(0), l(0)\} S_{G}\right]^{\frac{N}{2}}$. Hence,

$$
\nu(\bar{\Omega}) \geq \sum_{j \in J} \nu_{j} \geq \sum_{j \in J}\left(\min \{m(0), l(0)\} S_{G}\right)^{N / 2}
$$

and we infer from $\nu(\bar{\Omega})<+\infty$ that $J$ is finite.

In order to prove (2.10), we first use Hölder's inequality to compute

$$
\left|A_{n, \varepsilon}\right| \leq \int_{\Omega}\left|u_{n}\right|\left|\nabla u_{n}\right|\left|\nabla \phi_{\varepsilon}\right| \leq C\left(\int_{\Omega}\left|u_{n}\right|^{2}\left|\nabla \phi_{\varepsilon}\right|^{2}\right)^{1 / 2} .
$$

Since $u_{n} \rightarrow u$ strongly in $L^{2}(\Omega)$, the change of variables $y:=\left(x-x_{j}\right) / \varepsilon$ and the Sobolev embedding provide

$$
\begin{aligned}
\limsup _{n \rightarrow \infty}\left|A_{n, \varepsilon}\right| & \leq C\left(\int_{\Omega}|u|^{2}\left|\nabla \phi_{\varepsilon}\right|^{2}\right)^{1 / 2} \\
& =\varepsilon^{(N-2) / 2} C\left(\int_{\{|y| \leq \varepsilon\}}\left|u\left(y \varepsilon+x_{j}\right)\right|^{2}|\nabla \phi(y)|^{2} \mathrm{~d} y\right)^{1 / 2},
\end{aligned}
$$

and the first result follows from $N \geq 3$. The proof for $B_{n, \varepsilon}$ is analogous.

\section{The Palais-Smale condition}

Given a functional $I \in C^{1}(H, \mathbf{R})$ and $c \in \mathbf{R}$, we say that $I$ satisfies the PalaisSmale condition at level $c\left((P S)_{c}\right.$ for short) if any sequence $\left(z_{n}\right) \subset H$ such that

$$
\lim _{n \rightarrow+\infty} I\left(z_{n}\right)=c, \quad \lim _{n \rightarrow+\infty} I^{\prime}\left(z_{n}\right)=0
$$

has a convergent subsequence. We devote this section to the proof of the following local compactness result:

Proposition 3.1. If

$$
c^{*}:=\left(\frac{1}{\theta}-\frac{1}{2^{*}}\right)\left(\min \{m(0), l(0)\} S_{G}\right)^{N / 2},
$$

then $I_{a, b, \lambda}$ satisfies $(P S)_{c}$ for any $c<c^{*}$. 
Proof. Let $\left(z_{n}\right) \subset H$ be such that $I_{a, b, \lambda}^{\prime}\left(z_{n}\right) \rightarrow 0$ and $I_{a, b, \lambda}\left(z_{n}\right) \rightarrow c<c^{*}$. We first notice that, by $\left(m_{0}\right),\left(l_{0}\right),\left(F_{2}\right),(2.4)$ and $(2.2)$, there exists $C_{1}>0$ such that

$$
\begin{aligned}
c+o_{n}(1)\left\|z_{n}\right\|+o_{n}(1)= & I_{a, b, \lambda}\left(z_{n}\right)-\frac{1}{\theta}\left\langle I_{a, b, \lambda}^{\prime}\left(z_{n}\right), z_{n}\right\rangle \\
\geq & \frac{1}{2} M_{a}\left(\left\|u_{n}\right\|^{2}\right)+\frac{1}{2} L_{b}\left(\left\|v_{n}\right\|^{2}\right) \\
& -\frac{1}{\theta} m_{a}\left(\left\|u_{n}\right\|^{2}\right)\left\|u_{n}\right\|^{2}-\frac{1}{\theta} l_{b}\left(\left\|v_{n}\right\|^{2}\right)\left\|v_{n}\right\|^{2} \\
\geq & \left(\frac{1}{2} m(0)-\frac{1}{\theta} a\right)\left\|u_{n}\right\|^{2}+\left(\frac{1}{2} l(0)-\frac{1}{\theta} b\right)\left\|v_{n}\right\|^{2} . \\
\geq & C_{1}\left\|z_{n}\right\|^{2},
\end{aligned}
$$

and therefore $\left(z_{n}\right)$ is bounded in $H$.

Let $J$ be given by Lemma 2.3. We claim that $J=\varnothing$. Indeed, suppose by contradiction that there exists $j \in J$ and consider $\phi_{\varepsilon}$ as in the proof of Lemma 2.4. The boudedness of $\left(z_{n}\right),\left(F_{2}\right),(2.2),(2.4), \phi_{\varepsilon} \leq 1$, and the same argument used above provide

$$
c \geq\left(\frac{1}{\theta}-\frac{1}{2^{*}}\right) \int_{\Omega} G\left(u_{n}, v_{n}\right)+o_{n}(1) \geq\left(\frac{1}{\theta}-\frac{1}{2^{*}}\right) \int_{\Omega} \phi_{\varepsilon} G\left(u_{n}, v_{n}\right)+o_{n}(1) .
$$

Taking the limit and using (2.7), we obtain

$$
c \geq\left(\frac{1}{\theta}-\frac{1}{2^{*}}\right)\left(\min \{m(0), l(0)\} S_{G}\right)^{N / 2}=c^{*},
$$

contrary to $c<c^{*}$.

Since $J$ is empty, we infer from Lemma 2.3 that

$$
\lim _{n \rightarrow+\infty} \int_{\Omega} G\left(u_{n}, v_{n}\right)=\int_{\Omega} G(u, v) .
$$

Up to a subsequence, we have that

$$
\begin{cases}\left(u_{n}, v_{n}\right) \rightarrow(u, v) & \text { weakly in } H, \\ u_{n} \rightarrow u, v_{n} \rightarrow v, & \text { strongly in } L^{s}(\Omega), 2 \leq s<2^{*}, \\ u_{n}(x) \rightarrow u(x), v_{n}(x) \rightarrow v(x), & \text { a.e. in } \Omega, \\ \max \left\{\left|u_{n}(x)\right|,\left|v_{n}(x)\right|\right\} \leq g_{s}(x), & \text { a.e. in } \Omega,\end{cases}
$$

with $g_{s} \in L^{s}(\Omega), 2 \leq s<2^{*}$. By using $\left(F_{0}\right),\left(F_{1}\right),\left(F_{3}\right)$ and the strong convergence in $L^{s}(\Omega)$ we get

$$
\lim _{n \rightarrow+\infty} \int_{\Omega} z_{n} \cdot \nabla F\left(x, z_{n}\right)=\int_{\Omega} z \cdot \nabla F(x, z),
$$

where $z=(u, v)$. This and $\left\langle I_{a, b, \lambda}^{\prime}\left(z_{n}\right), z_{n}\right\rangle \rightarrow 0$ provide

$$
\lim _{n \rightarrow \infty}\left[m_{a}\left(\left\|u_{n}\right\|^{2}\right)\left\|u_{n}\right\|^{2}+l_{b}\left(\left\|v_{n}\right\|^{2}\right)\left\|v_{n}\right\|^{2}\right]=\lambda \int_{\Omega} z \cdot \nabla F(x, z)+\int_{\Omega} G(u, v) .
$$

On the other hand, since $\left\langle I_{a, b, \lambda}^{\prime}\left(z_{n}\right), z\right\rangle \rightarrow 0$, there holds

$$
m_{a}\left(\left\|u_{n}\right\|^{2}\right) \int_{\Omega}\left(\nabla u_{n} \cdot \nabla u\right)+l_{b}\left(\left\|v_{n}\right\|^{2}\right) \int_{\Omega}\left(\nabla v_{n} \cdot \nabla v\right)=A_{n}^{1}+A_{n}^{2}+o_{n}(1),
$$

where

$$
A_{n}^{1}:=\lambda \int_{\Omega} z \cdot \nabla F\left(x, z_{n}\right), \quad A_{n}^{2}:=\frac{1}{2^{*}} \int_{\Omega}(u, v) \cdot \nabla G\left(u_{n}, v_{n}\right) .
$$


Again, Lebesgue Theorem implies that

$$
\lim _{n \rightarrow+\infty} \int_{\Omega} z \cdot \nabla F\left(x, z_{n}\right)=\int_{\Omega} z \cdot \nabla F(x, z) .
$$

Furthermore, since the partial derivatives of $G$ are $\left(2^{*}-1\right)$-homogeneous, it follows from (2.5) that

$$
\begin{aligned}
\int_{\Omega}\left|G_{u}\left(u_{n}, v_{n}\right)\right|^{2^{*} /\left(2^{*}-1\right)} & \leq M_{G}^{2^{*} /\left(2^{*}-1\right)} \int_{\Omega}\left(\left|u_{n}\right|^{2^{*}-1}+\left|v_{n}\right|^{2^{*}-1}\right)^{2^{*} / 2^{*}-1} \\
& \leq C_{2} \int_{\Omega}\left(\left|u_{n}\right|^{2^{*}}+\left|v_{n}\right|^{2^{*}}\right)
\end{aligned}
$$

and therefore $\left(G_{u}\left(u_{n}, v_{n}\right)\right)$ is bounded in $L^{2^{*} /\left(2^{*}-1\right)}(\Omega)$. The pointwise convergence of $\left(u_{n}\right)$ and $\left(v_{n}\right)$ imply that $G\left(u_{n}, v_{n}\right) \rightarrow G(u, v)$ weakly in $L^{2^{*} /\left(2^{*}-1\right)}(\Omega)$. Since $u \in\left(L^{2^{*} / 2^{*}-1}(\Omega)\right)^{\prime}=L^{2^{*}}(\Omega)$, we obtain

$$
\lim _{n \rightarrow+\infty} \int_{\Omega} u G_{u}\left(u_{n}, v_{n}\right)=\int_{\Omega} u G_{u}(u, v)
$$

Analogously, $\int_{\Omega} v G_{v}\left(u_{n}, v_{n}\right) \rightarrow \int_{\Omega} v G_{v}(u, v)$, as $n \rightarrow+\infty$. The above considerations and (2.4) prove that

$$
\lim _{n \rightarrow+\infty} \int_{\Omega}(u, v) \cdot \nabla G\left(u_{n}, v_{n}\right)=\int_{\Omega}(u, v) \cdot \nabla G(u, v)=2^{*} \int_{\Omega} G(u, v) .
$$

If we define

$$
\alpha_{0}:=\lim _{n \rightarrow \infty}\left\|u_{n}\right\|, \quad \alpha_{1}:=\lim _{n \rightarrow \infty}\left\|v_{n}\right\|,
$$

we can take the limit in (3.2) and use the continuity of $m_{a}$ and $l_{b}$ to get

$$
m_{a}\left(\alpha_{0}^{2}\right)\|u\|^{2}+l_{b}\left(\alpha_{1}^{2}\right)\|v\|^{2}=\lambda \int_{\Omega} z \cdot \nabla F(x, z)+\int_{\Omega} G(u, v) .
$$

This and (3.1) imply that

$$
m_{a}\left(\alpha_{0}^{2}\right) \alpha_{0}^{2}+l_{b}\left(\alpha_{1}^{2}\right) \alpha_{1}^{2}=m_{a}\left(\alpha_{0}^{2}\right)\|u\|^{2}+l_{b}\left(\alpha_{1}^{2}\right)\|v\|^{2} .
$$

From the weak convergence of $\left(v_{n}\right)$, we know that $\|v\|^{2} \leq \alpha_{1}^{2}$. Hence,

$$
m_{a}\left(\alpha_{0}^{2}\right) \alpha_{0}^{2}+l_{b}\left(\alpha_{1}^{2}\right) \alpha_{1}^{2}=m_{a}\left(\alpha_{0}^{2}\right)\|u\|^{2}+l_{b}\left(\alpha_{1}^{2}\right)\|v\|^{2} \leq m_{a}\left(\alpha_{0}^{2}\right)\|u\|^{2}+l_{b}\left(\alpha_{1}^{2}\right) \alpha_{1}^{2},
$$

that is

$$
m_{a}\left(\alpha_{0}^{2}\right) \alpha_{0}^{2} \leq m_{a}\left(\alpha_{0}^{2}\right)\|u\|^{2} .
$$

Since $m_{a}\left(\alpha_{0}^{2}\right)>0$, this implies that $\alpha_{0}^{2} \leq\|u\|^{2} \leq \alpha_{0}^{2}$, and therefore $\left\|u_{n}\right\| \rightarrow \alpha_{0}$. It follows from the weak convergence of $\left(u_{n}\right)$ that $u_{n} \rightarrow u$ strongly in $H_{0}^{1}(\Omega)$. In the same way, $v_{n} \rightarrow v$ strongly in $H_{0}^{1}(\Omega)$. This concludes the proof.

\section{Proof of Theorem 1.1}

We start by checking that $I_{a, b, \lambda}$ satisfies the geometric conditions of the Mountain Pass Theorem.

Lemma 4.1. Suppose that $G$ satisfies $\left(G_{1}\right)$. Then,

(i) there eixst $\rho>0, \alpha>0$ such that

$$
I_{a, b, \lambda}(u, v) \geq \alpha>0, \quad \forall(u, v) \in H \cap \partial B_{\rho}(0) ;
$$

(ii) there exists $e \in H$, independent of $\lambda>0$, such that $I_{a, b, \lambda}(e)<0$. 
Proof. For any given $\varepsilon>0$, we can use $\left(F_{0}\right),\left(F_{1}\right)$ and $\left(F_{3}\right)$ to get

$$
F(x, s, t) \leq \frac{\varepsilon}{2}\left(|s|^{2}+|t|^{2}\right)+C\left(|t|^{q}+|s|^{q}\right), \quad \forall x \in \Omega,(s, t) \in \mathbf{R}^{2} .
$$

Picking $\varepsilon>0$ small, we can use the above inequality, $\left(m_{0}\right),\left(l_{0}\right)$ and $(2.5)$ to get

$$
\begin{aligned}
I_{a, b, \lambda}(u, v) \geq & \frac{1}{2} m(0)\|u\|^{2}+\frac{1}{2} l(0)\|v\|^{2}-\frac{\varepsilon}{2} \int_{\Omega}\left(|u|^{2}+|v|^{2}\right) \\
& -C_{1} \lambda \int_{\Omega}\left(|u|^{q}+|v|^{q}\right)-\frac{1}{2^{*}} \int_{\Omega} G(u, v) . \\
\geq & \|(u, v)\|^{2}\left(C_{2}-\lambda C_{3}\|(u, v)\|^{q-2}-C_{3}\|(u, v)\|^{2^{*}-2}\right) .
\end{aligned}
$$

Since $q \in\left(2,2^{*}\right)$, it is sufficient to choose $\rho>0$ sufficiently small to obtain (i).

In order to prove (ii), we consider $u_{0} \in H_{0}^{1}(\Omega)$ such that $\left\|u_{0}\right\|=1$ and $u_{0} \geq 0$. By $\left(F_{2}\right)$, we have that $F \geq 0$, and therefore

$$
\left.I_{a, b, \lambda}\left(t u_{0}, t u_{0}\right)\right) \leq a t^{2}+b t^{2}-\frac{t^{2^{*}}}{2^{*}} \int_{\Omega} G\left(u_{0}, u_{0}\right) .
$$

It follows from $\left(G_{1}\right)$ that $\int_{\Omega} G\left(u_{0}, u_{0}\right)>0$. Thus, $I_{a, b, \lambda}\left(t u_{0}, t u_{0}\right) \rightarrow-\infty$ as $t \rightarrow+\infty$ and (ii) holds for $e=t_{*}\left(u_{0}, u_{0}\right)$, with $t_{*}>0$ sufficiently large.

In view of the above result we obtain a sequence $\left(z_{n}\right) \subset H$ such that

$$
I_{a, b, \lambda}\left(z_{n}\right) \rightarrow c_{a, b, \lambda}, \quad I_{a, b, \lambda}^{\prime}\left(z_{n}\right) \rightarrow 0
$$

where

$$
c_{a, b, \lambda}:=\inf _{\gamma \in \Gamma} \max _{t \in[0,1]} I_{a, b, \lambda}(\gamma(t))>0
$$

and

$$
\Gamma=\left\{\gamma \in C([0,1], H): \gamma(0)=0 \text { and } I_{a, b, \lambda}(\gamma(1))<0\right\} .
$$

Lemma 4.2. The minimax level defined above satisfies

$$
\lim _{\lambda \rightarrow \infty} c_{a, b, \lambda}=0 \text {. }
$$

Proof. Let $u_{0} \in H_{0}^{1}(\Omega)$ be such that $\left\|u_{0}\right\|=1$ and $u_{0} \geq 0$. For $z_{0}:=\left(u_{0}, u_{0}\right)$, we set $\phi(t):=I_{a, b, \lambda}\left(t z_{0}\right)$, that is,

$$
\phi(t):=I_{a, b, \lambda}\left(t z_{0}\right)=\frac{1}{2} M_{a}\left(t^{2}\right)+\frac{1}{2} L_{b}\left(t^{2}\right)-\lambda \int_{\Omega} F\left(x, t z_{0}\right)-\frac{1}{2^{*}} \int_{\Omega} G\left(t z_{0}\right) .
$$

Clearly $I_{a, b, \lambda}(0)=0$. Moreover, from the proof of Lemma 4.1 we get $I_{a, b, \lambda}\left(\rho z_{0} /\left\|z_{0}\right\|\right)$ $>0$ and $I_{a, b, \lambda}\left(t z_{0}\right)<0$, for any $t>0$ large. Thus, there exists $t_{\lambda}>0$ such that

$$
I_{a, b, \lambda}\left(t_{\lambda} z_{0}\right)=\max _{t \geq 0} I_{a, b, \lambda}\left(z_{0}\right) .
$$

We claim that $t_{\lambda} \rightarrow 0$ as $\lambda \rightarrow+\infty$. Indeed, since $\phi^{\prime}\left(t_{\lambda}\right) t_{\lambda}=0$, we get

$$
t_{\lambda}^{2}\left(m_{a}\left(t_{\lambda}^{2}\right)+l_{b}\left(t_{\lambda}^{2}\right)\right)=\lambda \int_{\Omega}\left(t_{\lambda} z_{0}\right) \cdot \nabla F\left(x, t_{\lambda} z_{0}\right)+t_{\lambda}^{2^{*}} \int_{\Omega} G\left(u_{0}, u_{0}\right)
$$

and it follows from $\left(F_{2}\right),\left(G_{0}\right)$ and the definition of $m_{a}$ and $l_{b}$ that

$$
t_{\lambda}^{2^{*}-2} \int_{\Omega} G\left(u_{0}, u_{0}\right) \leq a+b
$$

which implies that $\left(t_{\lambda}\right)$ is bounded. Let $\left(\lambda_{n}\right) \subset \mathbf{R}$ be such that $\lambda_{n} \rightarrow+\infty$ and suppose that

$$
t^{*}:=\lim _{n \rightarrow \infty} t_{\lambda_{n}} \geq 0
$$


Since

$$
\lim _{\lambda \rightarrow+\infty} \int_{\Omega}\left(t_{\lambda} z_{0}\right) \cdot \nabla F\left(x, t_{\lambda} z_{0}\right)=\int_{\Omega}\left(t^{*} z_{0}\right) \cdot \nabla F\left(x, t^{*} z_{0}\right),
$$

we infer from (4.1) that $t^{*}=0$.

We now consider the path $\gamma_{*}(t)=t e, t \in[0,1]$, where $e \in H$ comes from Lemma 4.1. Notice that

$$
0<c_{a, b, \lambda} \leq \max _{t \in[0,1]} I\left(\gamma_{*}(t)\right)=I\left(t_{\lambda} z_{0}\right) \leq \frac{1}{2} M_{a}\left(t_{\lambda}^{2}\right)+\frac{1}{2} L_{b}\left(t_{\lambda}^{2}\right) .
$$

Taking $\lambda \rightarrow+\infty$ and recalling that $t_{\lambda} \rightarrow 0$, we conclude that $c_{a, b, \lambda_{n}} \rightarrow 0$

We are ready to prove our existence theorem.

Proof of Theorem 1.1. By the previous lemma there exists $\lambda_{0}>0$ such that

$$
c_{a, b, \lambda}<\left(\frac{1}{\theta}-\frac{1}{2^{*}}\right)\left(\min \{m(0), l(0)\} S_{G}\right)^{N / 2}, \quad \forall \lambda \geq \lambda_{0} .
$$

For each $\lambda>\lambda_{0}$, we can use Proposition 3.1 and the Mountain Pass Theorem to obtain $z_{\lambda}=\left(u_{\lambda}, v_{\lambda}\right) \in H$ such that $I_{a, b, \lambda}\left(z_{\lambda}\right)=c_{a, b, \lambda}>0$ and $I_{a, b, \lambda}^{\prime}\left(z_{\lambda}\right)=0$, that is, $z_{\lambda}$ is a nonnegative solution of the modified problem $\left(\widehat{S_{\lambda}}\right)$. According to Lemma 2.2 , it remains to check that, if $\lambda$ is large, then $\|u\| \leq s_{0}$ and $\|v\| \leq s_{1}$. Arguing by contradiction, suppose that there exists a sequence $\left(\lambda_{n}\right) \subset \mathbf{R}$ such that $\lambda_{n} \rightarrow+\infty$ and the respective solutions $z_{\lambda_{n}}=\left(u_{\lambda_{n}}, v_{\lambda_{n}}\right)$ verifies $\left\|u_{\lambda_{n}}\right\|>s_{0}$ or $\left\|v_{\lambda_{n}}\right\|>s_{1}$. By using $\left(F_{2}\right),(2.4)$ and $\left(G_{1}\right)$ we obtain

$$
\begin{aligned}
c_{a, b, \lambda_{n}} & =I_{a, b, \lambda}\left(u_{\lambda_{n}}, v_{\lambda_{n}}\right)-\frac{1}{\theta}\left\langle I_{a, b, \lambda_{n}}^{\prime}\left(z_{\lambda_{n}}\right), z_{\lambda_{n}}\right\rangle \\
& \geq\left(\frac{1}{2} m(0)-\frac{a}{\theta}\right) s_{0}^{2}+\left(\frac{1}{2} l(0)+\frac{b}{\theta}\right) s_{1}^{2},
\end{aligned}
$$

which contradicts $c_{a, b, \lambda_{n}} \rightarrow 0$. Hence, there exists $\lambda^{*}>0$ such that, for any $\lambda>\lambda^{*}$, the solution $\left(u_{\lambda}, v_{\lambda}\right)$ obtained above satisfies the problem $\left(S_{\lambda}\right)$. The same argument shows that $\left\|\left(u_{\lambda_{n}}, v_{\lambda_{n}}\right)\right\| \rightarrow 0$ as $\lambda_{n} \rightarrow+\infty$ and the theorem is proved.

\section{Proof of Theorem 1.2}

In this final section we prove our multiplicity result. Beside the basic assumptions $\left(m_{0}\right),\left(l_{0}\right),\left(F_{0}\right)-\left(F_{3}\right)$ and $\left(G_{0}\right)$, we shall suppose in this section that $\left(F_{5}\right),\left(\widehat{G_{1}}\right)$ and $\left(G_{3}\right)$ hold. As before, the weak solutions of the problem are critical points of

$$
I_{a, b, \lambda}(u, v):=\frac{1}{2} M_{a}\left(\|u\|^{2}\right)+\frac{1}{2} L_{b}\left(\|v\|^{2}\right)-\lambda \int_{\Omega} F(x, u, v)-\frac{1}{2^{*}} \int_{\Omega} G(u, v) .
$$

In order to obtain the critical points we shall apply the following version of the Symmetric Mountain Pass Theorem (see [2, 21]).

Theorem 5.1. Let $E=V \oplus W$ be a real Banach space with $\operatorname{dim} V<\infty$. Suppose that $I \in C^{1}(E, \mathbf{R})$ is an even functional satisfying $I(0)=0$ and

$\left(I_{1}\right)$ there exist $\rho, \alpha>0$ such that

$$
\inf _{u \in \partial B_{\rho}(0) \cap W} I(u) \geq \alpha ;
$$

$\left(I_{2}\right)$ there exists a subspace $\widehat{V} \subset E$ with $\operatorname{dim} V<\operatorname{dim} \widehat{V}<\infty$ such that, for some $M>0$

$$
\max _{u \in \widehat{V}} I(u) \leq M
$$


$\left(I_{3}\right) I$ satisfies the $(P S)_{c}$ for any $c \in(0, M)$.

Them $I$ possesses at least $(\operatorname{dim} \widehat{V}-\operatorname{dim} V)$ pairs of nonzero critical points.

The same argument used in the proof of Proposition 3.1 provides the local compactness result below:

Proposition 5.2. If

$$
c_{*}:=\min \left\{c^{*},\left(\frac{m(0)}{2}-\frac{a}{\theta}\right) s_{0}^{2},\left(\frac{l(0)}{2}-\frac{b}{\theta}\right) s_{1}^{2}\right\},
$$

then $I_{a, b, \lambda}$ satisfies $(P S)_{c}$ for any $c<c_{*}$.

We prove below the geometric condition $\left(I_{2}\right)$.

Proposition 5.3. Suppose that $G$ satisfies $\left(\widehat{G_{1}}\right)$. Then, for each $k \in \mathbf{N}$ and $M_{*}>0$, there exist $\lambda^{*}>0$ with the following property: for any $\lambda \geq \lambda^{*}$ we can find a subspace $V_{k}^{\lambda} \subset H$ such that $\operatorname{dim} V_{k}^{\lambda}=k$ and

$$
\sup _{z \in V_{k}^{\lambda}} I_{a, b, \lambda}(z)<M_{*} \text {. }
$$

Proof. Let $\varphi \in C_{0}^{\infty}\left(B_{1}(0)\right)$ and fix $\left\{x_{1}, \ldots, x_{m}\right\} \subset \Omega$ and $\delta>0$ in such way that, for $i, j \in I:=\{1, \ldots, m\}, B_{\delta}\left(x_{i}\right) \subset \Omega$ and $B_{\delta}\left(x_{i}\right) \cap B_{\delta}\left(x_{j}\right)=\varnothing$, if $i \neq j$. For each $i \in I$, we set $\varphi_{i}^{\delta}(x):=\varphi\left(\left(x-x_{i}\right) / \delta\right)$ and use the change of variables $y:=\left(x-x_{i}\right) / \delta$ to compute

$$
A_{\delta}:=\frac{\left\|\varphi_{i}^{\delta}\right\|^{2}}{\left\|\varphi_{i}^{\delta}\right\|_{\theta}^{2}}=\frac{\int_{\Omega}\left|\nabla \varphi\left(\left(x-x_{i}\right) / \delta\right)\right|^{2}}{\left(\int_{\Omega}\left|\varphi\left(\left(x-x_{i}\right) / \delta\right)\right|^{\theta}\right)^{2 / \theta}}=\frac{\int_{\Omega} \frac{\delta^{N}}{\delta^{2}}|\nabla \varphi(y)|^{2} \mathrm{~d} y}{\left(\int_{\Omega} \delta^{N}|\varphi(y)|^{\theta} \mathrm{d} y\right)^{2 / \theta}}
$$

that is

$$
A_{\delta}=\delta^{[N-2-(2 N / \delta)]} \frac{\|\varphi\|^{2}}{\|\varphi\|_{\theta}^{2}} .
$$

Since $\mathbf{R}^{m}$ has finite dimension, there exists $d_{1}=d_{1}(k, \theta)$ such that

$$
\sum_{i=1}^{k}\left|y_{i}\right|^{\theta} \geq d_{1}\left(\sum_{i=1}^{k}\left|y_{i}\right|^{2}\right)^{\theta / 2}, \quad \forall\left(y_{1}, \ldots, y_{m}\right) \in \mathbf{R}^{k} .
$$

Set

$$
V_{k, \delta}:=\operatorname{span}\left\{\left(\varphi_{1}^{\delta}, 0\right), \ldots,\left(\varphi_{k}^{\delta}, 0\right)\right\}
$$

and consider $u=\sum_{i=1}^{k} \alpha_{i} \varphi_{i}^{\delta} \in V_{k, \delta}$. By using (5.1), (5.2) and the fact that the support of the functions $\varphi_{i}^{\delta}$ are disjoints, we obtain

$$
\begin{aligned}
\int_{\Omega}|u|^{\theta} & =\int_{B_{\delta}\left(x_{1}\right) \cup \cdots \cup B_{\delta}\left(x_{k}\right)}\left|\sum_{i=1}^{k} \alpha_{i} \varphi_{i}^{\delta}\right|^{\theta} \mathrm{d} x=\sum_{i=1}^{k}\left\|\alpha_{i} \varphi_{i}^{\delta}\right\|_{\theta}^{\theta} \\
& \geq d_{1}\left(\sum_{i=1}^{k}\left\|\alpha_{i} \varphi_{i}^{\delta}\right\|_{\theta}^{2}\right)^{\theta / 2}=c_{1}\left(\sum_{i=1}^{k} A_{\delta}^{-1}\left\|\alpha_{i} \varphi_{i}^{\delta}\right\|^{2}\right)^{\theta / 2}=d_{2} \delta^{\gamma}\|u\|^{\theta},
\end{aligned}
$$

where $d_{2}:=d_{1}\|\varphi\|^{2}\|\varphi\|_{q}^{-2}>0$ and

$$
\gamma:=-\left(N-2-\frac{2 \theta}{N}\right) \frac{\theta}{2}>0
$$


Integrating the inequality in $\left(F_{2}\right)$ we get

$$
F(x, s, 0) \geq d_{3}|s|^{\theta}-d_{4}, \quad \forall(x, s) \in \Omega \times \mathbf{R},
$$

and therefore can use (5.3) to get, for any $z=(u, 0) \in V_{k, \delta}$,

$$
\begin{aligned}
I_{a, b, \lambda}(z) & \leq \frac{a}{2}\|u\|^{2}-\lambda \sum_{i=1}^{m} \int_{B_{\delta}\left(x_{i}\right)} F(x, u, 0) \mathrm{d} x \\
& \leq \frac{a}{2}\|u\|^{2}-\lambda d_{2} d_{3} \delta^{\gamma}\|u\|^{\theta}-\lambda d_{2} k \delta^{N} \omega_{N},
\end{aligned}
$$

where $\omega_{N}$ is the volume of the unitary ball. Hence, for some positive constants $d_{5}=d_{5}(k, \theta), d_{6}=d_{6}(k, N)$ we have that

$$
I_{a, b, \lambda}(z) \leq \frac{a}{2}\|u\|^{2}-\lambda d_{5} \delta^{\gamma}\|u\|^{\theta}+\lambda d_{6} \delta^{N}, \quad \forall z=(u, 0) \in V_{k, \delta} .
$$

Since $\theta<2^{*}$, we have that $\gamma<N$ and therefore we can pick $\gamma_{0} \in(\gamma, N)$ and consider the function

$$
h_{\delta}(t):=\frac{a}{2} t^{2}-d_{5} \delta^{-\gamma_{0}+\gamma} t^{\theta}+d_{6} \delta^{-\gamma_{0}+N}, \quad t>0 .
$$

which attains its maximun value at $t_{\delta}:=\left[a\left(d_{5} \theta\right)^{-1} \delta^{\gamma-\gamma_{0}}\right]^{1 /(\theta-2)}$. This and $\gamma_{0} \in(\gamma, N)$ imply that $h_{\delta}\left(t_{\delta}\right) \rightarrow 0$ as $\delta \rightarrow 0^{+}$. So, there exists $\delta^{*}=\delta^{*}(k, \theta, N, a)>0$ such that

$$
\max _{t \geq 0} h_{\delta}(t)<\frac{M_{*}}{2}, \quad \forall \delta \in\left(0, \delta^{*}\right]
$$

We now set $\lambda_{k}^{*}:=\left(\delta^{*}\right)^{-\gamma_{0}}$. Let $\lambda \geq \lambda_{k}^{*}$ and define the $k$-dimensional subspace $V_{k}^{\lambda}:=V_{k, \delta}$, for $\delta=\lambda^{-1 / \gamma_{0}}$. Since $\delta^{-\gamma_{0}}=\lambda \geq \lambda_{k}^{*}=\left(\delta^{*}\right)^{-\gamma_{0}}$, we obtain $\delta \leq \delta^{*}$. Hence, for any $z=(u, 0) \in V_{k}^{\lambda}$, we can use (5.4) and the above inequality to get

$$
I_{a, b, \lambda}(z) \leq \frac{a}{2}\|u\|^{2}-\delta^{-\gamma_{0}} d_{5} \delta^{\gamma}\|u\|^{\theta}+\delta^{-\gamma_{0}} d_{6} \delta^{N} \leq \max _{t \geq 0} h_{\delta}(t)<\frac{M_{*}}{2},
$$

and we have done.

Proof of Theorem 1.2. Given $k \in \mathbf{N}$, we are intending to apply Theorem 5.1 with $W=H$. The condition $\left(I_{1}\right)$ can be proved as in Lemma 4.1. If we consider $M_{*}<c_{*}$ as in the statement of Proposition 5.2, it remains to obtain a subspace of dimension $k$ such that $\left(I_{2}\right)$ holds. However, this condition is always true for the subspace $V_{k}^{\lambda}$ given in Proposition 5.3, provide we consider $\lambda \geq \lambda_{k}^{*}$. Since $I_{a, b, \lambda}(0)=0$ and the functional is even, the hypotheses of Theorem 5.1 are satisfied and therefore, for each $\lambda \geq \lambda_{k}^{*}$, there exist $k$ pairs of nonzero solutions for the modified problem $\left(\widehat{S_{\lambda}}\right)$.

Let $z=(u, v) \in H$ be one of the solutions obtained above. Since $I_{a, b, \lambda}(z) \leq$ $M_{*} \leq c_{*}$, we can use the definition of $c_{*},\left(F_{2}\right),(2.4)$ and $\left(\widehat{G_{1}}\right)$ to get

$$
\left(\frac{1}{2} m(0)-\frac{a}{\theta}\right)\|u\|^{2} \leq I_{a, b, \lambda}(z)-\frac{1}{\theta}\left\langle I_{a, b, \lambda}^{\prime}(z), z\right\rangle \leq M_{*}<c_{*} \leq\left(\frac{1}{2} m(0)-\frac{a}{\theta}\right) s_{0}^{2},
$$

from which it follows that $\|u\|<s_{0}$. The same argument provides $\|v\| \leq s_{1}$ and therefore, as in the proof of Lemma 2.2, we conclude that $z$ is a nonzero solution of $\left(S_{\lambda}\right)$. 


\section{References}

[1] Alves, C. O., F. J.S. A. CorrêA, and T. F. MA: Positive solutions for a quasilinear elliptic equation of Kirchhoff type. - Comput. Math. Appl. 49, 2005, 85-93.

[2] Ambrosetti, A., and P. H. Rabinowitz: Dual variational methods in critical point theory and applications. - J. Funct. Anal. 14, 1973 349-381.

[3] CorrêA, F. J.S. A., and R. G. Nascimento: On a nonlocal elliptic system of $p$-Kirchhoff type under Neumann boundary condition. - Math. Comp. Model. 49, 2009, 598-604.

[4] Eisley, G.: Nonlinear vibrations of beams and rectangular plates. - Z. Angew. Math. Phys. 15, 1964, 167-175.

[5] Figueiredo, G. M.: On a nonlocal system with critical growth. - Recent Trends on Nonlinear Elliptic Systems, International Scientific Press, Athens, Greece, 2012.

[6] Figueiredo, G. M.: Existence of a positive solution for a Kirchhoff problem type with critical growth via truncation argument. - J. Math. Anal. Appl. 401, 2013, 706-713.

[7] Figueiredo, G. M., and J. R. S. Junior: Multiplicity of solutions for a Kirchhoff equation with subcritical and critical growth. - Diff. Int. Equations 25, 2012, 853-868.

[8] Furtado, M. F., L. D. Oliveira, and J. P. P. DA Silva: Multiple solutions for a Kirchhoff equation with critical growth. - Z. Angew. Math. Phys. 70, 2019, Article 11.

[9] Hamydy, A., M. Massar, and N. Tsouli: Existence of solutions for p-Kirchhoff type problems with critical exponent. - Electron. J. Differential Equations 2011, No. 105, 1-8.

[10] He, X. M., and W. M. Zou: Infinitely many positive solutions for Kirchhoff-type problems. Nonlinear Anal. 70, 2009, 1407-1414.

[11] Jin, J., and X. WU: Infinitely many radial solutions for Kirchhoff-type problems in $\mathbf{R}^{N}$. - J. Math. Anal. Appl. 369, 2010, 564-574.

[12] Kirchhoff, G.: Vorlesungen über Mathematische Physik: Mechanik. - Teubner, Leipzig, 1876.

[13] Li, L., and X. ZHong: Infinitely many small solutions for the Kirchhoff equation with local sublinear nonlinearities. - J. Math. Anal. Appl. 435, 2016, 955-967.

[14] Limaco, J., and L. A. Medeiros: Kirchhoff-Carrier elastic strings in noncylindrical domains. - Portugaliae Mathematica 14, 1999, 464-500.

[15] LiOns, J. L.: On some questions in boundary value problems of mathematical physics. - In: International Symposium on Continuum, Mechanics and Partial Differential Equations, Rio de Janeiro (1977), Mathematics Studies 30, North-Holland, Amsterdam, 1978, 284-346.

[16] Lions, P. L.: The concentration-compactness principle in the calculus of variations, Part I. Rev. Mat. Iberoamericana 1, 1985, 145-201

[17] LiU, D. C.: On a $p$-Kirchhoff equation via fountain theorem and dual fountain theorem. Nonlinear Anal. 72, 2010, 208-302.

[18] MaO, A. M., and J. T. Zhang: Sign-changing and multiple solutions of Kirchhoff type problems without the P.S. condition. - Nonlinear Anal. 70, 2009, 1275-1287.

[19] De Morais Filho, D. C., and M. A.S. Souto: Systems of $p$-Laplacean equations involving homogeneous nonlinearities with critical Sobolev exponent degrees. - Comm. Partial Differential Equations 24, 1999, 1537-1553.

[20] NAimen, D.: Positive solutions of Kirchhoff type elliptic equations involving a critical Sobolev exponent. - NoDEA Nonlinear Differential Equations Appl. 21, 2014, 885-914.

[21] Silva, E. A. B., and M. S. XAVIER: Multiplicity of solutions for quasilinear elliptic problems involving critical Sobolev exponents. - Ann. Inst. H. Poincaré Anal. Non Linéaire 20, 2003, $341-358$.

[22] Sun, J., and C. TANG: Existence and multiplicity of solutions for Kirchhoff type equations. Nonlinear Anal. 74, 2011, 1212-1222. 